\title{
Garden of Eden states in traffic models
}

\author{
Andreas Schadschneider ${ }^{1}$ and Michael Schreckenberg ${ }^{2}$ \\ ${ }^{1}$ Institut für Theoretische Physik \\ Universität zu Köln \\ D-50937 Köln, Germany \\ email: as@thp.uni-koeln.de \\ 2 Theoretische Physik/FB 10 \\ Gerhard-Mercator-Universität Duisburg \\ D-47048 Duisburg, Germany \\ email: schreck@uni-duisburg.de
}

May 7, 2018

\begin{abstract}
We investigate the allowed configurations in the stationary state of the cellular automaton model for single-lane traffic. It is found that certain states in the configuration space can not be reached if one uses parallel dynamics. These so-called Garden of Eden (GoE) states do not exist for random-sequential dynamics and are responsible for the strong short-ranged correlations found in parallel dynamics. By eliminating the GoE states we obtain a simple and effective approximative description of the model. For $v_{\max }=1$ the exact solution is recovered. For $v_{\max }=2$ this elimination leads to much higher values of the flux compared to the mean-field result which are in good agreement with Monte Carlo simulations.
\end{abstract}




\section{Introduction}

The description of traffic flow using cellular automata (CA) is quite successful [1], despite the simplicity of the model [2]. CA are, by design, ideal for large-scale computer simulations. This fact has already been used for the simulation of urban traffic in various cities, see e.g. [3, 4, 5]. On the other hand, analytical descriptions are difficult. In [6, 7, 8] we have developed several methods which yield an approximate description of the stationary state. In certain limits (e.g. $v_{\max }=1$ or $p \rightarrow 0$ ), these methods even become exact (see [9] for a review). These approaches are based on a microscopic description which takes into account certain correlations. Part of the difficulties come from the fact that one uses parallel dynamics. This introduces a non-local aspect into the problem since the whole lattice is updated at once. On the other hand, random-sequential dynamics is much simpler to treat analytically. For $v_{\max }=1$ for instance, simple mean-field theory gives already the correct steady state, i.e. there are no correlations. Here we propose a rather simple analytical approach which exhibits the main difference between parallel and random-sequential dynamics very clearly. For parallel dynamics not all states of the configuration space can be reached by the dynamics, some are 'dynamically forbidden'. This is not the case for random-sequential dynamics.

For completeness we recall the definition of the CA model for single-lane traffic [2]. The street is divided into $L$ cells which can be occupied by at most one car or be empty. The state of each car is described by an internal parameter ('velocity') which can take on only integer values $v=0,1,2, \ldots, v_{\max }$. The state of the system at time $t+1$ can be obtained from the state at the previous time $t$ by applying the following

four simple update steps to all cars at the same time (parallel dynamics):

R1 Acceleration: $v_{j}(t) \rightarrow v_{j}\left(t+\frac{1}{3}\right)=\min \left\{v_{j}(t)+1, v_{\max }\right\}$

R2 Braking: if $v_{j}\left(t+\frac{1}{3}\right)>d_{j}(t)$ then $v_{j}\left(t+\frac{1}{3}\right) \rightarrow v_{j}\left(t+\frac{2}{3}\right)=d_{j}(t)$

R3 Randomization: $v_{j}\left(t+\frac{2}{3}\right) \stackrel{p}{\rightarrow} v_{j}(t+1)=\max \left\{0, v_{j}\left(t+\frac{2}{3}\right)-1\right\}$ with probability $p$

R4 Driving: car $j$ moves $v_{j}(t+1)$ cells.

Here $d_{j}(t)$ denotes the number of empty cells in front of car $j$, i.e. the gap or headway. In the following it will be important that $v_{j}(t)$ is just the number of cells that car $j$ moved in the timestep $t-1 \rightarrow t$.

In [7] we investigated a microscopic mean-field theory (MFT) for this kind of cellular automaton. The most important result was that the flows obtained are much too small compared to computer simulations. The reason is that MFT cannot account for the 'particle-hole attraction' found in the stationary state, i.e. the probability to find an empty cell in front of a (moving) car is enhanced compared to the mean-field 
result. This effect is taken into account by the $n$-cluster approximation introduced in [6, 7]. Here the lattice is divided into clusters of length $n$ which overlap $n-1$ cellsi]. It turned out that the 2-cluster approximation is exact for $v_{\max }=1$. For $v_{\max }=2$ already small cluster sizes (e.g. $n=4$ ) gave an excellent agreement with numerical results for the flow.

In [8] an alternative analytic approach was introduced, the so-called car-oriented mean-field theory (COMF). Here the dynamical variables are not the occupancies of the cells but the gaps $d_{j}(t)$ between consecutive cars. In the COMF these gaps are treated as being independent. Again we found that COMF is exact for $v_{\max }=1$. For $v_{\max }=2$ and the 'traditional' value $p=0.5$ the flow obtained from COMF is comparable to the 3 -cluster result. However, the COMF seems to become exact in the limit $p \rightarrow 0$, in contrast to the 2-cluster approach [9].

In the present paper we like to present a rather simple extension of MFT. The key idea is a reduction of the configuration space by removing all states which cannot by reached dynamically. In the context of cellular automata these states are called Garden of Eden (GoE) states or paradisical states. A simple example (see Fig. 1) for $v_{\text {max }}=2$ is the configuration $(\bullet, 1,2)$ of two consecutive cells, where ' $\bullet$ denotes an empty cell and the numbers correspond to the velocities of the cars. Cars move from left to right. Obviously the velocity is just the number of cells the car moved in the previous timestep. Therefore the configuration $(\bullet, 1,2)$ could have evolved only from a state which has two cars in the leftmost cell. Since double-occupations are not allowed in the present model, states containing $(\bullet, 1,2)$ are dynamically forbidden, i.e. are GoE states.

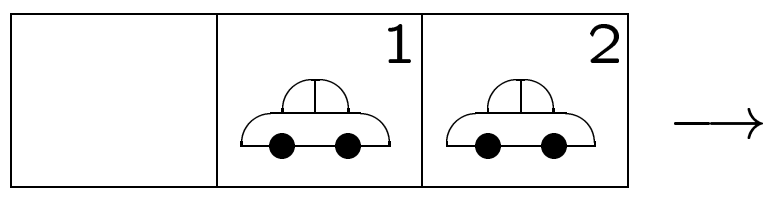

Figure 1: A Garden of Eden state for the model with $v_{\max } \geq 2$.

Here we reinvestigate MFT for $v_{\max }=1$ and $v_{\max }=2$, but eliminate the GoE states. This paradisical mean-field ( $p M F)$ theory will lead to a considerable improvement of the results.

\section{Mean-field theory}

Here we briefly review the MFT results for $v_{\max }=1$ and $v_{\max }=2$. A more complete account along with a detailed derivation can be found in [7]. The densities of cars

\footnotetext{
${ }^{\dagger}$ MFT correponds to $n=1$.
} 
with velocity $v\left(v=0,1, \ldots, v_{\max }\right)$ in the stationary state is denoted by $c_{v}$. Therefore the full density of cars is given by $c=\sum_{v=0}^{v_{\max }} c_{v}$. For convenience we also introduce the 'hole' density $d=1-c$ and the abbreviation $q=1-p$. The flux (or current) is given by $f(c)=\sum_{v=1}^{v_{\max }} v c_{v}$.

For $v_{\max }=1$ the mean-field equations then read:

$$
\begin{aligned}
& c_{0}=(c+p d) c \\
& c_{1}=q c d
\end{aligned}
$$

so that the flux is simply given by

$$
f_{M F}^{(1)}(c)=c_{1}=q c(1-c)
$$

For $v_{\max }=2$ the rate equations for the densities are given by

$$
\begin{aligned}
c_{0} & =(c+p d) c_{0}+(1+p d) c\left(c_{1}+c_{2}\right), \\
c_{1} & =d\left[q c_{0}+(q c+p d)\left(c_{1}+c_{2}\right)\right] \\
c_{2} & =q d^{2}\left(c_{1}+c_{2}\right) .
\end{aligned}
$$

The solution

$$
\begin{aligned}
c_{0} & =\frac{(1+p d) c^{2}}{1-p d^{2}}, \\
c_{1} & =\frac{q\left(1-q d^{2}\right) d c}{1-p d^{2}}, \\
c_{2} & =\frac{q^{2} d^{3} c}{1-p d^{2}}
\end{aligned}
$$

yields for the flux

$$
f_{M F}^{(2)}(c)=c_{1}+2 c_{2}=\frac{q\left(1+q d^{2}\right) d c}{1-p d^{2}} .
$$

Comparison with the results from Monte Carlo simulations shows that the MFT results underestimate the flow considerably [7]. Therefore strong short-range correlations exist which increase the flow compared to the prediction of MFT. Using the exact solution for the case $v_{\max }=1$ [6] one can demonstrate explicitely the existance of a strong particle-hole attraction, i.e. the probability to find an empty cell in front of an occupied cell is enhanced compared to the MFT result.

At first sight, this result is surprising. For random-sequential dynamics it is known [2] that MFT is exact for $v_{\max }=1$. Therefore the origin of the correlations is the parallel update procedure. In the following we will see that the existence of the GoE states is responsible for the differences between parallel and random-sequential dynamics.

\footnotetext{
${ }^{\ddagger}$ In random-sequential dynamics in each timestep a cell which is updated is picked at random.
} 


\section{GoE states for $v_{\max }=1$}

The question, whether a state is a GoE state or not, can be decided locally by investigating just nearest-neighbour configurations. It turns out that GoE states are all states containing the local configurations $(0,1)$ or $(1,1)$, i.e. a moving vehicle is directly followed by another car. This is not possible as can be seen by looking at the possible configurations at the previous timestep. The momentary velocity gives the number of cells that the car moved in the previous timestep. In both configurations the first car moved one cell. Therefore it is immediately clear that $(0,1)$ is a GoE state since otherwise there would have been a doubly-occupied cell before the last timestep. The configuration $(1,1)$ is also not possible since both cars must have occupied neighbouring cells before the last timestep too. Therefore, according to rule $\mathrm{R} 2$, the second car could not move.

We now modify the MFT equations (11) and (2) to take into account the existence of GoE states. Following the procedure described in [7] it turns out that only (1) has to be modified. Due to this modification the normalization $c_{0}+c_{1}=c$ is no longer satisfied automatically. Therefore a normalization constant $\mathcal{N}$ has to be introduced. The final equations are than given by

$$
\begin{aligned}
& c_{0}=\mathcal{N}\left(c_{0}+p d\right) c, \\
& c_{1}=\mathcal{N} q c d,
\end{aligned}
$$

with the normalization

$$
\mathcal{N}=\frac{1}{c_{0}+d}
$$

Since $c_{0}+c_{1}=c$ we have only one independent variable for fixed density $c$, e.g. $c_{1}$. Solving (11), (12) for $c_{1}$ we obtain

$$
c_{1}=\frac{1}{2}(1-\sqrt{1-4 q(1-c) c}) .
$$

The flow is given by $f(c)=c_{1}$ and we recover the exact solution for the case $v_{\max }=1$ [6].

This result confirms the expectations mentioned above. One can see clearly that the difference between random-sequential and parallel dynamics is the existence of GoE states in the latter. After eliminating these GoE states, no correlations are left in the reduced configuration space.

\section{GoE states for $v_{\max }=2$}

In this case more GoE states exist. In order to identify the GoE states it is helpful to note that the rules R1-R4 imply $d_{j}(t)=d_{j}(t-1)+v_{j+1}(t)-v_{j}(t)$ and therefore

$$
d_{j}(t) \geq v_{j+1}(t)-v_{j}(t)
$$




$$
v_{j}(t) \leq d_{j}(t-1)
$$

The second inequality (16) is a consequence of R2.

In the following we list the elementary GoE states, i.e. the local configurations which are dynamically forbidden (cars move from left to right):

$$
\begin{array}{ccc}
(0,1), \quad(0,2), \quad(1,2), & (0, \bullet, 2), \\
(1,1), \quad(2,1), \quad(2,2), \quad(1, \bullet, 2), & (2, \bullet, 2), \\
& (0, \bullet, \bullet, 2) .
\end{array}
$$

The numbers give the velocity of a vehicle in an occupied cell and • denotes an empty cell. Using Monte Carlo simulations we have checked that there are no further elementary GoE states for clusters up to 10 cells.

The elementary GoE states in (17) violate the inequality (15), and the configurations in (18) violate (16). The state in (19) is a second order GoE state. Going one step back in time leads to a first order GoE state since $(0, \bullet, \bullet, 2)$ must have evolved from $(0, v)$ (with $v=1$ or $v=2$ ).

Again we can derive the pMF equations by modifying the method for the derivation of the MF mean-field theory [7]. Taking into account only the first order GoE states (17) and (18) one obtains the following pMF equations:

$$
\begin{aligned}
& c_{0}=\mathcal{N}\left[c_{0} c+p d\left(c_{0}+c_{1} c\right)\right], \\
& c_{1}=\mathcal{N}\left[p d^{2}\left(c_{1}+c_{2}\right)+q d\left(c_{0}+c_{1} c\right)\right], \\
& c_{2}=\mathcal{N} q d^{2}\left(c_{1}+c_{2}\right) .
\end{aligned}
$$

The normalization $\mathcal{N}$ ensures $c_{0}+c_{1}+c_{2}=c$ and is given explicitly by

$$
\mathcal{N}=\frac{1}{c_{0}+d c_{1}+d^{2} c_{2}}=\frac{1}{c_{0}+d\left(1-c_{2}\right)} .
$$

Using (22) we can express $c_{2}$ through $c_{0}$ and $c$ only:

$$
c_{2}=\frac{1}{2 d}\left(c_{0}+d-\sqrt{\left(c_{0}+d\right)^{2}-4 q d^{3}\left(c-c_{0}\right)}\right) .
$$

Inserting this result into (20) we obtain a cubic equation which determines $c_{0}$ in terms of the parameters $c$ and $p$ :

$$
\alpha c_{0}^{3}+\beta c_{0}^{2}+\gamma c_{0}+\delta=0
$$

where the coefficients are given by

$$
\begin{aligned}
\alpha & =-q d^{3}-p d^{2}+q d-q \\
\beta & =\left(3 p^{2}-p-1\right) d^{4}+\left(-p^{2}-3 p+1\right) d^{3}+\left(-p^{2}+p+2\right) d^{2}-3 q d+q \\
\gamma & =p c d\left[\left(-p^{2}-3 p+2\right) d^{3}+\left(p^{2}+p+1\right) d^{2}+(2 p-3) d+q\right] \\
\delta & =-(p c d)^{3} .
\end{aligned}
$$


In principle the zeroes of (25) can be determined explicitely. The physical solution is the one in the interval $[0,1]$. The flow can be calculated as $f(c, p)=c_{1}+2 c_{1}$ where $c_{1}$ and $c_{2}$ are determined by (24) and $c=c_{0}+c_{1}+c_{2}$.
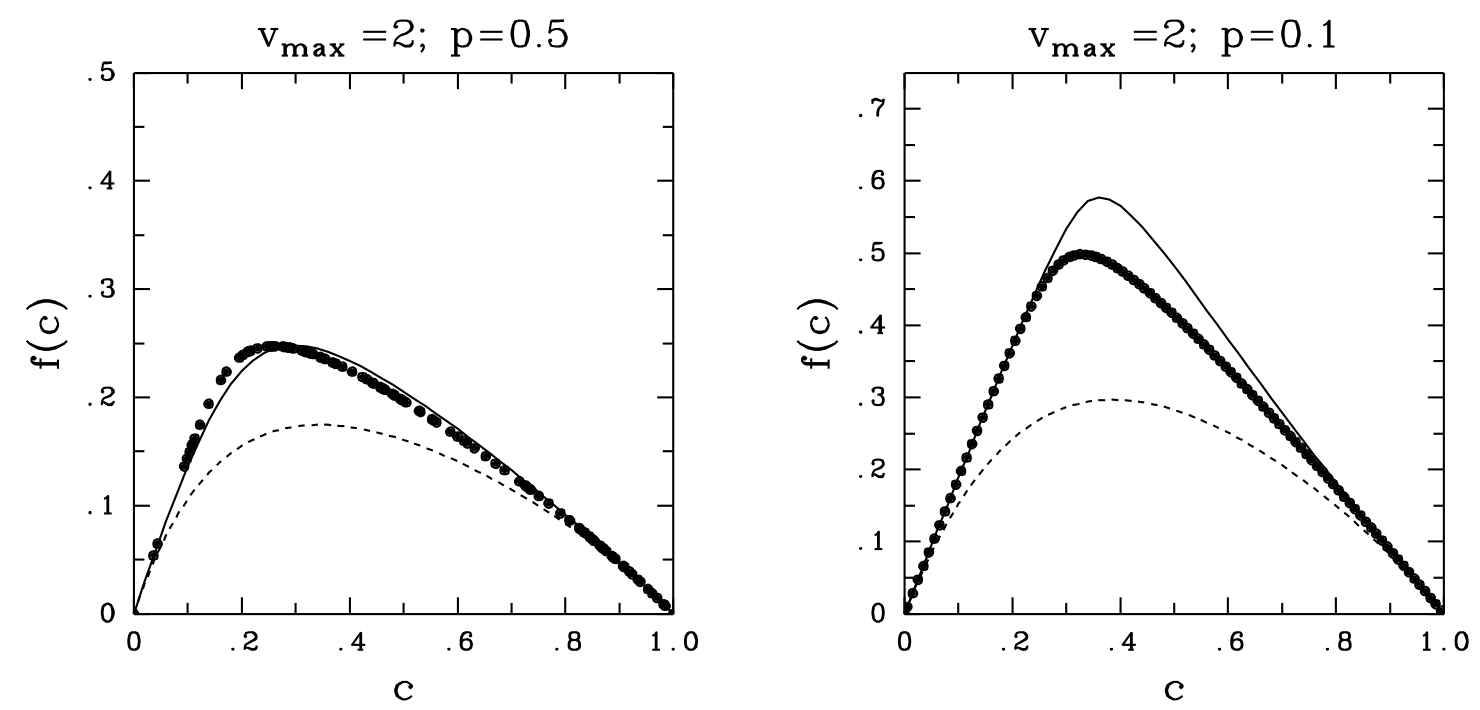

Figure 2: Fundamental diagram for $v_{\max }=2$ and $p=0.5$ (left) and $p=0.1$ (right). Comparison of paradisical MF (full line) with results from computer simulations (•) and the naive MF approximation (dotted line).

We have also calculated the pMF equations which in addition take into account the second order GoE state (19). One has to modify the equations for $c_{0}$ and $c_{1}$ only (and the normalization) by replacing the last term in (20) by $p d\left\{\left(c_{0}+c_{1}\right) c+c_{0} d\left(1-c_{2}\right)\right\}$ and the last term in (21) by $q d\left\{\left(c_{0}+c_{1}\right) c+c_{0} d\left(1-c_{2}\right)\right\}$. However, these modifications lead only to minor changes in the results for the fundamental diagram.

\section{Discussion}

We have presented an analysis of the allowed configurations in the CA model for traffic flow. Due to the use of parallel dynamics not all configurations can be reached through the dynamics. Eliminating these Garden of Eden states allowed us to improve the results of the naive mean-field theory considerably.

GoE states can be characterized locally. We identified all elementary GoE configurations for $v_{\max }=1$ and $v_{\max }=2$. It turns out that for $v_{\max }=1$ it is sufficient to investigate only configurations of all clusters of two cells. For $v_{\max }=2$ the largest elementary GoE configuration consists of four neighbouring cells.

For $v_{\text {max }}=1$ the paradisical mean-field theory is able to reproduce the exact solution. This implies that in the subspace without GoE states all configurations are equally 
probable. This has to be compared with random-sequential dynamics. Here all configurations are equally probable and naive mean-field theory is exact. This means that the strong short-ranged correlations found for parallel update are solely due to the use of parallel dynamics.

In fact one may speculate that this is rather general. The difference between randomsequential and parallel update comes mainly from the existence of GoE states in the latter. This implies that a method that 'works' for random-sequential dynamics (e.g. an exact solution or good approximation) should also work for parallel dynamics, but now in the subspace without GoE states.

For $v_{\max }=2$ the paradisical mean-field theory yields a considerable improvement of the mean-field results, but it does not become exact. One observes a qualitative difference to the case $v_{\max }=1$, since now there are correlations present which can not be explained by the existence of GoE states.

The existence of GoE states gives a simple criterion for the quality of an approximation: A good approximation should be able to account for all GoE states. This can be illustrated for the case $v_{\max }=1$. The methods used previously for the exact solution are the 2-cluster approach [6, 7] and Car-Oriented Mean-Field theory (COMF) [8]. Both methods are able to identify both GoE states $(0,1)$ and $(1,1)$. For

$v_{\max }=2$ one needs at least the 4-cluster approximation to account for all GoE states (17-19). Indeed, the results of [6, 7] show that the 4-cluster results are in excellent agreement with simulation results. COMF is able to identify all GoE states since all elementary GoE configurations consist of only two neighbouring cars, i.e. there are no elementary GoE states with three vehicles.

Finally we want to point out that the model is ergodic in the sense that for configurations $\tau, \tau^{\prime}$ which appear in the stationary state with probabilities $P(\tau), P\left(\tau^{\prime}\right)>0$ there is a non-vanishing transition probability $P\left(\tau \rightarrow \tau^{\prime}\right)$. The existence of GoE states poses no problems in computer simulations. If the initial state is a GoE state, it will become a non-GoE state after the first timestep.

The method presented here is also applicable to other models. An interesting case is the asymmetric exclusion process (see [10] and references therein), which is identically to the model investigated here with $v_{\max }=1$, but with open boundary conditions where an injection and/or removal of particles is possible. In [11] the existence of GoE states has been used to obtain an approximative description of the deterministic limit of the ASEP which is in excellent agreement with numerical results.

Acknowledgements: Part of this work has been performed within the research program of the Sonderforschungsbereich 341 (Köln-Aachen-Jülich).

\section{References}


[1] D.E. Wolf, M. Schreckenberg, A. Bachem (Eds.): Traffic and Granular Flow, World Scientific, Singapore (1996)

[2] K. Nagel, M. Schreckenberg: J. Phys. I France 2, 2221 (1992)

[3] J. Esser, M. Schreckenberg: Int. J. Mod. Phys. C8, 1025 (1997)

[4] M. Rickert, K. Nagel: Int. J. Mod. Phys. C8, 483 (1997)

[5] K. Nagel, C.L. Barrett: Int. J. Mod. Phys. C8, 505 (1997)

[6] A. Schadschneider, M. Schreckenberg: J. Phys. A26, L679 (1993)

[7] M. Schreckenberg, A. Schadschneider, K. Nagel, N. Ito: Phys. Rev. E51, 2939 (1995)

[8] A. Schadschneider, M. Schreckenberg: J. Phys. A30, L69 (1997)

[9] A. Schadschneider: cond-mat/9711296, to be published in Traffic and Granular Flow '97 (Eds.: M. Schreckenberg, D.E. Wolf), Springer (1998)

[10] N. Rajewsky, L. Santen, A. Schadschneider, M. Schreckenberg: condmat/9710316

[11] L.G. Tilstra, M.H. Ernst: cond-mat/9712237 